\title{
Preferential MGMT methylation could predispose a subset of KIT/PDGFRA-WT GISTs, including SDH-deficient ones, to respond to alkylating agents
}

Riccardo Ricci ${ }^{1,2^{*}}$ (D), Maurizio Martini ${ }^{1,2}$, Gloria Ravegnini ${ }^{3}$, Tonia Cenci ${ }^{2}$, Massimo Milione ${ }^{4}$, Paola Lanza ${ }^{2}$, Francesco Pierconti ${ }^{1,2}$, Donatella Santini ${ }^{5}$, Sabrina Angelini ${ }^{3}$, Alberto Biondi ${ }^{6}$, Fausto Rosa ${ }^{7}$, Sergio Alfieri ${ }^{7,8}$, Gennaro Clemente ${ }^{8,9}$, Roberto Persiani ${ }^{6,8}$, Alessandra Cassano ${ }^{10,11}$, Maria A. Pantaleo ${ }^{12}$ and Luigi M. Larocca ${ }^{1,2}$

\begin{abstract}
Background: Succinate dehydrogenase (SDH)-deficient gastrointestinal stromal tumors (GISTs) constitute a small KIT/ PDGFRA-WT GIST subgroup featuring DNA methylation which, although pervasive, appears nevertheless not randomly distributed. Although often indolent, these tumors are mostly chemorefractory in aggressive cases. Promoter methylation-induced $O^{6}$-methylguanine DNA methyltransferase (MGMT) inactivation improves the efficacy of alkylating agents in gliomas, colorectal cancer and diffuse large B cell lymphoma. MGMT methylation has been found in some GISTs, without determining SDH status. Thirty-six GISTs were enrolled in past sarcoma trials testing alkylating agents, with negative results. Nevertheless, a possible effect on MGMT-methylated GISTs could have escaped detection, since tested GISTs were neither selected by genotype nor investigated for SDH; MGMT was studied in two cases only, revealing baseline activity; these trials were performed prior to the adoption of Choi criteria, the most sensitive for detecting GIST responses to therapy. Under these circumstances, we investigated whether MGMT methylation is preferentially found in SDH-deficient cases (identified by SDHB immunohistochemistry) by analyzing 48 pathogenetically heterogeneous GISTs by methylation-specific PCR, as a premise for possible investigations on the use of alkylating drugs in these tumors.

Results: Nine GISTs of our series were SDH-deficient, revealing significantly enriched in MGMT-methylated cases (6/9-67\%-, vs. 6/39-15\%- of SDH-proficient GISTs; $p=0.004)$. The pathogenetically heterogeneous KIT/ PDGFRA-WT GISTs were also significantly MGMT-methylated (11/24-46\%-, vs. 1/24-4\%- of KIT/PDGFRA-mutant cases, $p=0.002$.

Conclusions: A subset of KIT/PDGFRA-WT GISTs, including their largest pathogenetically characterized subgroup (i.e., SDH-deficient ones), is preferentially MGMT-methylated. This finding could foster a reappraisal of alkylating agents for treating malignant cases occurring among these overall chemorefractory tumors.

Keywords: Gastrointestinal stromal tumors, Succinate dehydrogenase, $0^{6}$-methylguanine DNA methyltransferase, DNA methylation, Wild-type GIST, Molecular diagnosis
\end{abstract}

\footnotetext{
*Correspondence: riccardo.ricci@unicatt.it

'Department of Pathology, Università Cattolica del Sacro Cuore, Largo F.Vito

1, 00168 Rome, Italy

${ }^{2}$ UOC di Anatomia Patologica, Fondazione Policlinico Universitario "A.

Gemelli" IRCCS, Largo A. Gemelli 8, 00168 Rome, Italy

Full list of author information is available at the end of the article
}

(c) The Author(s). 2019 Open Access This article is distributed under the terms of the Creative Commons Attribution 4.0 International License (http://creativecommons.org/licenses/by/4.0/), which permits unrestricted use, distribution, and reproduction in any medium, provided you give appropriate credit to the original author(s) and the source, provide a link to the Creative Commons license, and indicate if changes were made. The Creative Commons Public Domain Dedication waiver (http://creativecommons.org/publicdomain/zero/1.0/) applies to the data made available in this article, unless otherwise stated. 


\section{Background}

Gastrointestinal (GI) stromal tumors (GISTs), the most common mesenchymal tumors of the GI tract, revealed a heterogeneous family of tumors differing in molecular trigger and, consequently, in pathogenesis, prognosis and therapy [1, 2]. Their vast majority hinges upon activating mutations of either KIT or platelet-derived growth factor receptor alpha (PDGFRA), being often responsive to the tyrosin-kinase (TK)-inhibitor (TKI) imatinib. GISTs wild-type (WT) for these two genes, including their largest pathogenetically characterized subgroup (i.e., the succinate dehydrogenase (SDH)-deficient subset), although often indolent, may behave aggressively, being usually unresponsive to the TKI therapies commonly used [3-5]. In particular, presently, there is no specific evidence of an effective therapy for SDH-deficient GISTs with the possible exception of regorafenib and sunitinib for their anti-angiogenic mechanism of action, even though mature data are still lacking $[6,7]$.

The exploitation of reduced activity of $\mathrm{O}^{6}$-methylguanine DNA methyltransferase (MGMT) due to epigenetic silencing is a promising approach for selectively targeting tumors employing alkylating agents, with reduced host toxicity. This strategy has been shown to be effective in glioma, colorectal cancer and diffuse large B cell lymphoma [8-11].

SDH-deficient GISTs feature pervasive DNA methylation, which appears, nevertheless, to be not randomly distributed, as evidenced by the significant number of recurrent hypo- and hypermethylated genomic targets found in these tumors and by the hypermethylation of $S D H C$, typical of SDH-deficient GISTs WT for $S D H-A, B, C$, and $D$ genes (collectively termed $S D H x$ ) but not of $S D H x$-mutant ones [12-14]. Noticeably, although reported in a few GISTs [15-17], MGMT methylation has never been investigated in SDH-deficient cases. Trials on the use of alkylating agents temozolomide and carmustine in sarcomas evaluated 36 GISTs, with negative results [18-20]. However, these GISTs were neither selected by genotype nor, coherent with the epoch of the studies, selected and/or investigated for SDH. Moreover, MGMT was studied in two cases only, revealing baseline activity; the actual MGMT depletion following administration of $\mathrm{O}^{6}$-benzylguanine, a MGMT-inactivating substrate, was not verified [20]. Finally, these trials were performed prior to the adoption of Choi criteria, the most sensitive method for assessing GIST response to therapy [21]. A possible effect of alkylating agents on MGMT-methylated GISTs could thus have been missed, possibly due to an inappropriate selection of cases and a lack of proper evaluation of tumor response. Following these premises, we analyzed whether SDH-deficient GISTs are preferentially MGMT-methylated, as a precondition for possible investigations on the use of alkylating drugs in the treatment of these tumors.

\section{Methods \\ Study population}

Forty-eight GISTs, assorted so as to represent various pathogenetic types, were retrieved from the files of the pathology departments of the Catholic University of Rome and of the Sant'Orsola-Malpighi Hospital of Bologna, differing in anatomic site and morphology. All the cases had been previously characterized by hematoxylin/eosin staining of sections from formalinfixed, paraffin-embedded (FFPE) specimens; by immunohistochemistry (IHC) for CD117 and DOG1; and by genetic analysis of $K I T$ (exons 9, 11, 13, and 17) and PDGFRA (exons 12, 14, and 18). In cases WT for these genes, KRAS (exon 2) and BRAF (V600E) were also analyzed; previously reported protocols were followed [2224]. BRAF status was also analyzed in five KIT or PDGFRA-mutant cases (noticeably, BRAF mutations have been only exceptionally detected together with $K I T$ or PDGFRA ones [25]). Five GISTs (from three patients) arose in the context of neurofibromatosis type 1 (NF1). Tumor features are detailed in Table 1. Four cases, including two tumors previously characterized for SDH status, have been previously published [22, 26, 27].

\section{SDH analysis}

SDHB IHC was performed on all KIT and PDGFRA-WT cases, irrespective of site or morphology. Although GISTs depending on molecular triggers involving genes other than $S D H x$ have been consistently proved SDHB-positive [28], we investigated for SDHB expression fifteen of such tumors in our series as a control. Mouse monoclonal antibody to SDHB 21A11 (ABCAM, Cambridge, MA, 1:1000) was employed. The Leica BondMax autostainer (Leica Microsystems, Bannockburn, IL) was employed utilizing the BondMax avidin biotin-free polymer-based detection system preceded by heat-induced epitope retrieval with Leica retrieval solution (alkaline buffer), using diaminobenzine as the chromogen. Only slides with positive internal control (smooth muscle, endothelial, epithelial, or lymphoid cells) were considered for analysis.

Genetic analysis of $S D H$ subunits was performed in SDHB-negative cases as follows. The exons of the subunits of SDH complex were sequenced on tumor and normal tissue using the Sanger sequencing method on ABI 310 Genetic Analyzer (Applied Biosystems). DNA was extracted from tumor and normal FFPE specimens by the QIAmp DNA Micro kit (Qiagen, Milan, Italy) in accordance with manufacturer's recommendations. 


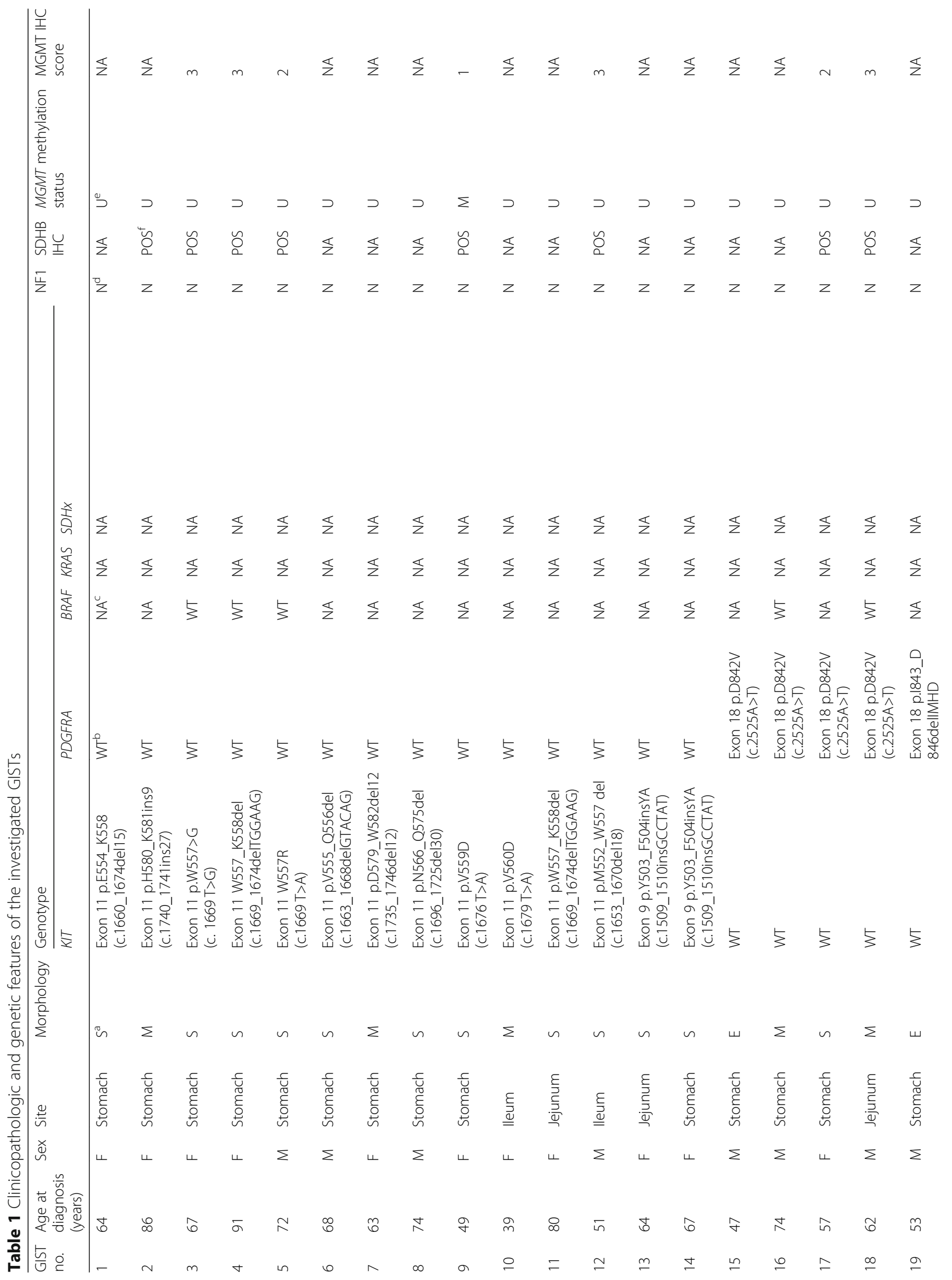




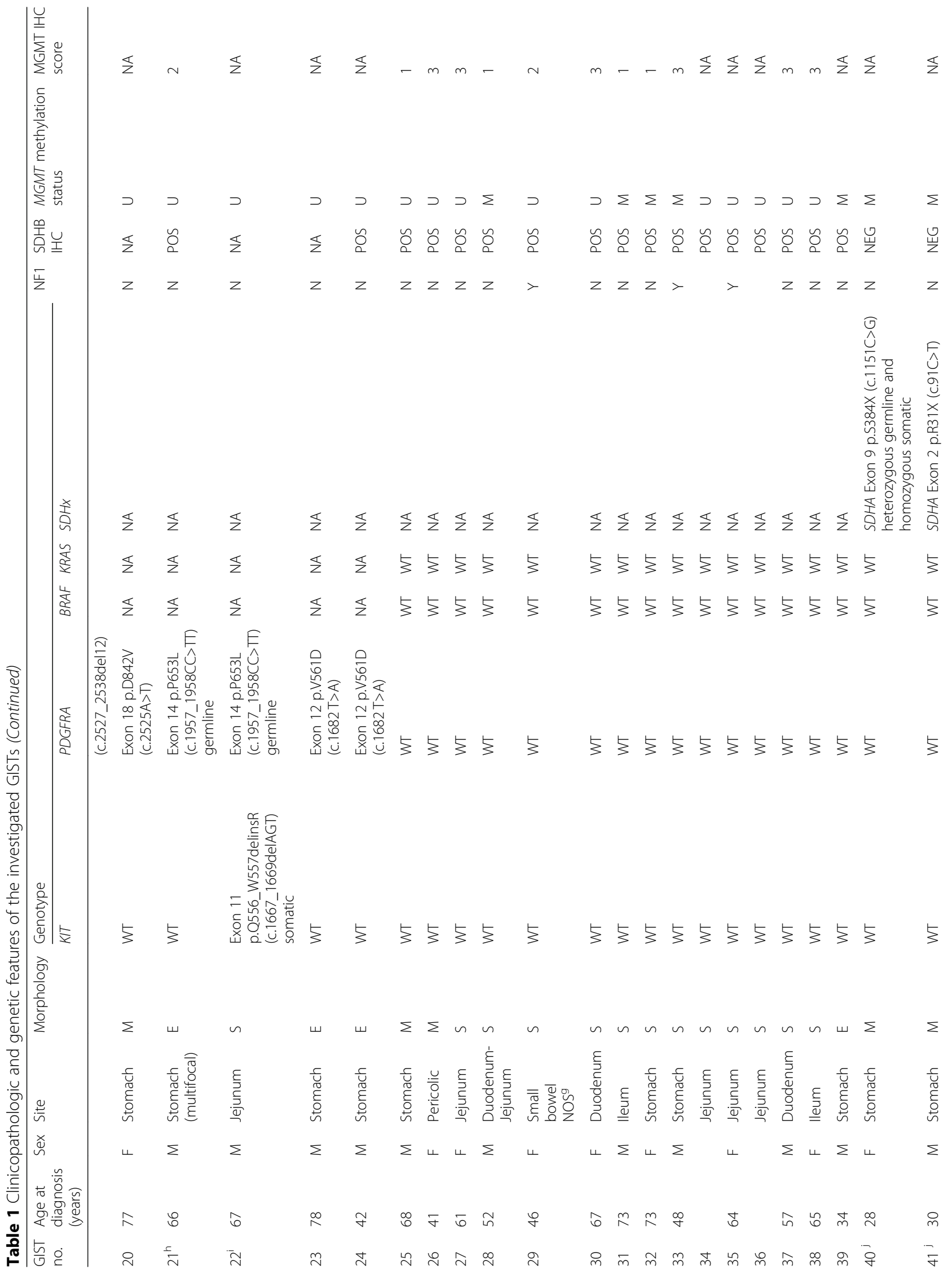


Ricci et al. Clinical Epigenetics $\quad$ (2019) 11:2

Page 5 of 11

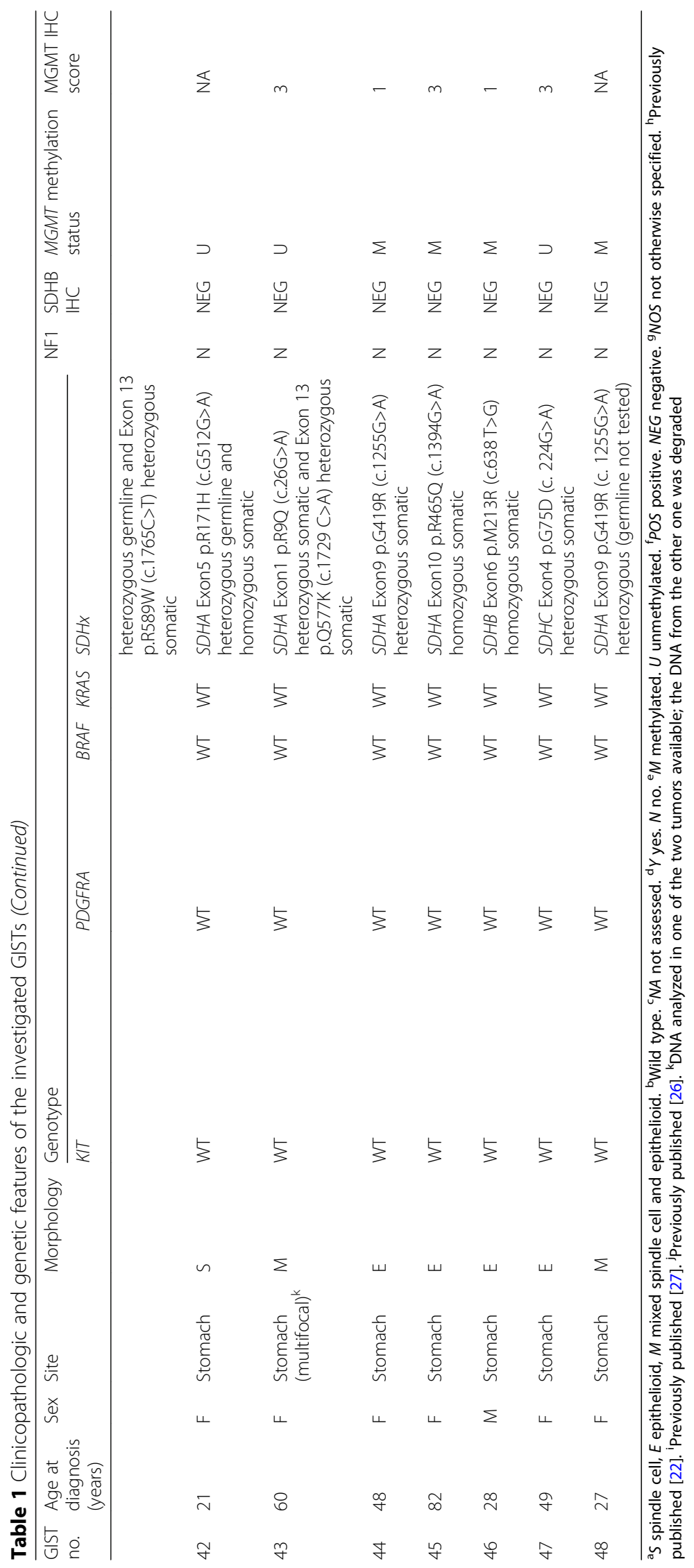


Briefly, FFPE slices (three 10- $\mu$ m-thick slices for each sample) were digested overnight at $56{ }^{\circ} \mathrm{C}$ in ATL buffer with the addition of proteinase K (Qiagen). DNA extraction was then continued with QIAamp DNA micro kit (Qiagen). Each exon was amplified with polymerase chain reaction (PCR) amplification using specific primer pairs, as previously reported [29]. PCR was carried out in a total volume of $25 \mu \mathrm{l}$ consisting in $20 \mathrm{ng}$ of DNA, $10 \times$ PCR buffer, $\mathrm{MgCl} 2$, dNTP, primers (10 pM each), and $1 \mathrm{U}$ FastStart DNA Taq polymerase (Roche). PCR conditions were an initial denaturation of $95^{\circ} \mathrm{C}$ for 5 min, followed by 40 cycles of $95^{\circ} \mathrm{C}$ for $30 \mathrm{~s}, 52-64{ }^{\circ} \mathrm{C}$ for $30 \mathrm{~s}$, and $72^{\circ} \mathrm{C}$ for $30 \mathrm{~s}$. PCR products were purified with the Qiaquick PCR purification kit (Qiagen) and sequenced on both strands using the Big Dye Terminator v1.1 Cycle Sequencing kit (Applied Biosystems).

\section{MGMT analysis}

DNA extraction and bisulfite modification were performed on three $10-\mu \mathrm{m}$ slides from paraffin-embedded tissues, as previously described [30]. The pathologic areas selected for DNA extraction contained at least 70\% disease-specific cells. The methylation status of the GpG islands in the promoter region of MGMT was determined as described elsewhere [31]. Briefly, bisulfitemodified DNA (100-200 ng) was amplified in a mixture containing $1 \times \mathrm{PCR}$ buffer $(20-\mathrm{mM}$ Tris [pH 8.3], $50-\mathrm{mM} \mathrm{KCl}, 1.5-\mathrm{mM} \mathrm{MgCl} 2)$, deoxynucleotide triphosphates $(0.2 \mathrm{mM}$ each), primers (20 pM each), and $0.75 \mathrm{U}$ GoTaq Hot Start polymerase (Promega, Madison, Wis) in a final volume of $25 \mu \mathrm{l}$. PCR conditions were an initial denaturation of $95^{\circ} \mathrm{C}$ for $8 \mathrm{~min}$, followed by 35 cycles of $95^{\circ} \mathrm{C}$ for $60 \mathrm{~s}, 60^{\circ} \mathrm{C}$ for $60 \mathrm{~s}$, and $72{ }^{\circ} \mathrm{C}$ for $60 \mathrm{~s}$. PCR products were electrophoresed in a $2.5 \%$ agarose gel, stained with ethidium bromide, and visualized under ultraviolet illumination (93 and $81 \mathrm{bp}$ were the lengths of the unmethylated and methylathed bands, respectively). Methylation-specific PCR (MS-PCR) analysis was performed in duplicate for all samples. Normal lymphocyte DNA supermethylated with SssI methyltransferase (New England Biolabs, Beverly, Mass) and treated with bisulfite was used as the unmethylated and methylated control, water as a negative control, and untreated DNA as internal PCR control.

For MGMT immunohistochemistry, mouse monoclonal antibody to MGMT MT 23.2 (Thermo Fisher, Waltham, MA, 1:2000) was employed. The Dako autostainer link 48 was employed utilizing the EnVision ${ }^{\text {mix }}$ FLEX+ detection system (Dako, Glostrup, Denmark) preceded by heat-induced epitope retrieval (EDTA $120^{\circ}, 10 \mathrm{~min}$ ). Only slides with positive internal control (endothelial, lymphoid, epithelial, or smooth muscle cells) were considered for analysis. MGMT expression was scored by two pathologists, blinded to tumor methylation status, molecular, and clinical data, using a three-tiered scale as previously reported (scores 1,2 , and 3 for $<10 \%$, 10 to $49 \%$, and $\geq 50 \%$ tumor cells featuring intensely and uniformly stained nuclei, respectively) [32].

\section{Statistical analysis}

Two-tailed Fisher's exact tests were performed to compare pairs of data sets using Statistica 12 software (Statsoft Inc., Tulsa, OK).

\section{Results \\ SDH analysis}

To identify functional loss of the SDH complex in GISTs, we performed SHDB immunohistochemistry [33]. Nine GISTs were SDHB-negative. All of them were gastric, non-NF1-related, at-least-in-part epithelioid (see Additional file 1: Figure S1, which shows a typical SDH-negative GIST from our series) and WT for KIT, PDGFRA, KRAS, and BRAF. All these GISTs harbored mutations in one of the subunits of $S D H$, namely, $S D H A$ in seven cases, $S D H B$ in one case, and $S D H C$ in one case. A somatic second hit flanked a germline mutation in three cases; in five tumors, the genetic alterations were exclusively somatic: in two cases, homozygous (likely due to loss of heterozygosis), in one case compound heterozygous, and in two cases heterozygous; in one case, the germline $S D H$ status could not be investigated (Table 1 and Additional file 2: Figure S2, which shows chromatograms of the $S D H x$ mutations found in cases $42-48$ of our series).

\section{MGMT analysis}

To ascertain whether MGMT promoter CpG islands were methylated in GISTs, and whether MGMT methylation pattern varied among the pathogenetically heterogeneous GIST subgroups of our series, we analyzed MGMT by MS-PCR. MGMT was methylated in only 1 out of 24 (4\%) KIT or PDGFRA-mutant GISTs (namely, an exon-11-KIT-mutant case), against $11 M G M T$-methylated cases of the remaining 24 (46\%) KIT/PDGFRA-WT GISTs $(p=0.002$, Fisher exact test). KRAS and BRAF were WT in all the evaluated cases of our series, including all the KIT/ PDGFRA WT tumors. Six out of $9(67 \%)$ and 6 out of 39 (15\%) cases revealed MGMT-methylated among SDHB-deficient and SDHB-proficient GISTs, respectively ( $p=0.004$, Fisher's exact test). MGMT was methylated in one out of the five NF1-related GISTs. Figure 1 is a representative of the MS-PCR results for MGMT promoter in some GISTs of our series. Data are resumed in Table 1.

To assess the impact of MGMT methylation on MGMT expression, we performed MGMT immunohistochemistry in half of GISTs of our series. The overall 


Fig. 1 Methylation status of $M G M T$ promoter gene (MS-PCR) in four examples of GIST. Lanes containing PCR products derived from unmethylated and
methylated alleles are marked $\mathrm{U}$ and $\mathrm{M}$, respectively (with a length of 93 and 81 bp, in that order). GISTs 41 and 44 show a hypermethylated MGMT
promoter gene; the same gene is unmethylated in GIST 10 and 17 (the bands present in the $\mathrm{M}$ lanes of these two GISTs and in both lanes of $\mathrm{UC}$ are
primer dimers). UC untreated control DNA, PC positive control DNA, MW molecular weight marks (100-bp ladder)

correlation between MGMT promoter methylation and MGMT immunohistochemical expression (score 1 vs. scores $2-3)$ was good ( $p=0.001$, Fisher's exact test), although a minority of tumors showed either a high-MGMT IHC score in the presence of MGMT methylation or the reverse condition (Table 1, Fig. 2).

\section{Discussion}

We herein assessed MGMT methylation status in a population of GISTs heterogeneous with respect to pathogenesis. Our results show that a subset of GISTs WT for KIT and PDGFRA, including their largest pathogenetically characterized subpopulation, i.e., the SDH-deficient ones, is significantly enriched in MGMT-methylated cases, with SDH-deficient GISTs featuring the highest prevalence of this tumor variant. Thus, these subsets of GISTs, characterized by a problematic clinical management in case of malignancy because of their usual resistance to imatinib and variable response to other drugs, appear potentially predisposed to respond to alkylating agents.

GISTs WT for KIT and PDGFRA constitute a pathogenetically heterogeneous tumor group accounting for about $10-15 \%$ of GISTs [1]. Taken together, these GISTs are characterized by an overall indolent behavior $[3,4]$. However, malignant cases may occur, often accompanied by unresponsiveness to the commonly used TKI therapies [5].

SDH-deficient GISTs, characterized by negativity at SDHB immunostaining, constitute the largest pathogenetically characterized subgroup among cases WT for KIT and PDGFRA. Their pathogenesis basically follows the classic tumor-suppressor gene model, with both alleles of one of the four $S D H$ subunits inactivated in the tumor cells, either on a mutational (often with a germline

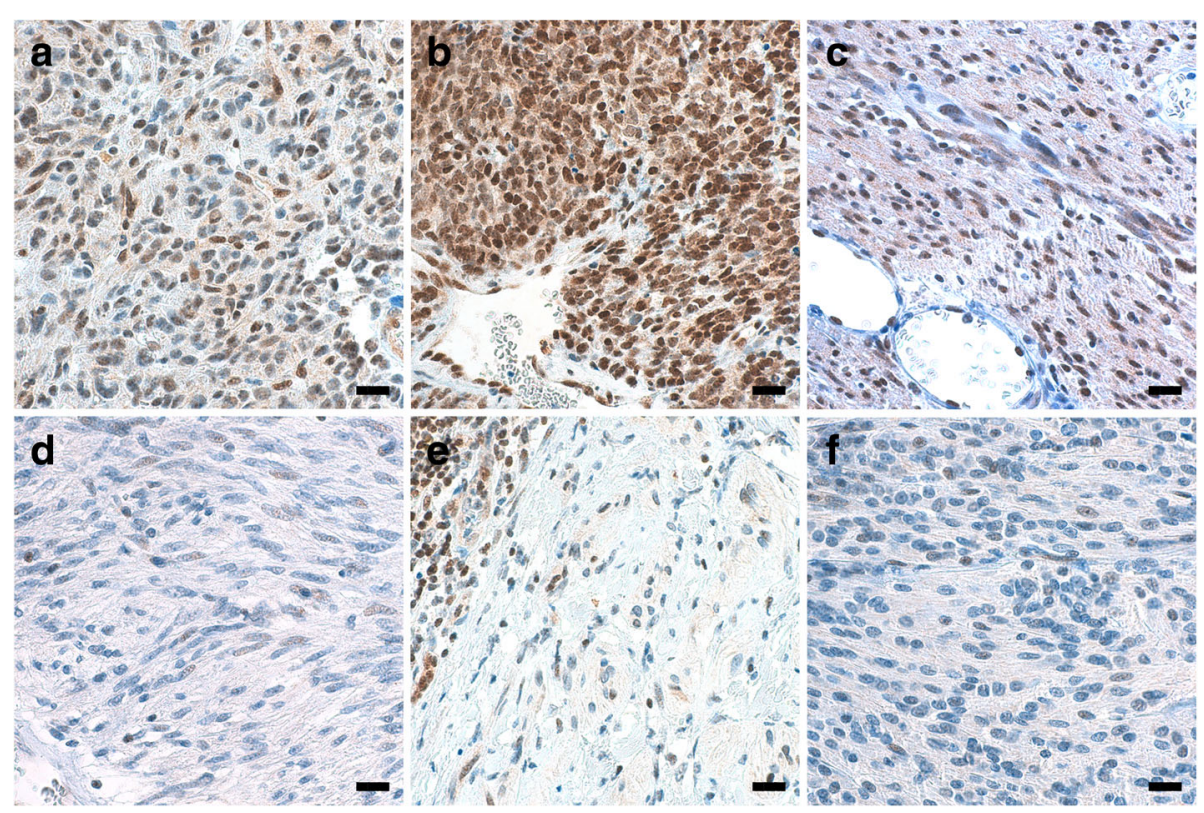

Fig. 2 MGMT protein expression assessed by IHC in six examples of GIST. At MGMT IHC, MGMT-unmethylated GISTs 43 (a), 26 (b), and 27 (c) featured nuclear staining in the majority of cells (score 3), unlike MGMT-methylated GISTs 32 (d), 31 (e), and 44 (f) (score 1). Endothelial and lymphoid cells (the periphery of a lymphoid aggregate is evident in e, top left) act as internal positive controls. (Scale bars: $20 \mu \mathrm{m}$ ) 
component) or epigenetic (SDHC hypermethylation) base, possibly presenting in syndromic settings (Carney triad or Carney-Stratakis syndromes) [3, 12, 28, 34]. Their progression can be extremely slow, with reported time lapses between primary tumor and metastasis as long as 42 years [33], and their stage and clinical behavior are often incoherent, with frequent prolonged survival in the presence of metastases. These features, likely caused by the metabolic disadvantage due to SDH deficiency, can make the attribution of disease stability to a drug effect problematic [7]. Nevertheless, SDH-deficient GIST can behave aggressively, with an overall mortality probably higher than $15 \%$, with cases fatal in a few years [28].

Imatinib, a TKI acting on several KIT and PDGFRA isoforms, proved ineffective in SDH-deficient GISTs [28], coherently with SDH deficiency being a trigger independent of KIT/PDGFRA. No mature data concerning the use of sunitinib in these neoplasms are available, although a clinical benefit due to an anti-angiogenic mechanism may occur. Of note, the rarity of SDH-deficient GISTs makes investigations on pure populations of such tumors very difficult. Consequently, clues for establishing an effective chemotherapy for this GIST subset are often inferred from studies on KIT/PDGFRA-WT cases, which are enriched in SDH-deficient tumors. Following these premises, drugs of potential interest for treating the latter neoplasms, in part represented by experimental molecules based on their biology, include linsitinib, PI3K/AKT/mTOR inhibitors, nilotinib, sorafenib, heat-shock protein inhibitors, chemicals targeting hypoxia-inducible factor- $1 \alpha$, and demethylating agents such as decitabine [14, 35-39]. Nevertheless, we presently do not dispose of a reliably effective drug for treating SDH-deficient GISTs, with the possible exception of regorafenib, recently reported to induce objective responses and durable benefit [6].

$M G M T$ inactivation through gene methylation allows the effective employment of alkylating agents in heterogeneous human tumors [8-11]. SDH-deficient GISTs display DNA methylation (both in the presence of $S D H x$ mutations or not) which, although overall pervasive, is not randomly distributed [12-14]. We therefore studied MGMT methylation in the SDH-deficient GIST subgroup, as a premise to possible investigations on the employment of alkylating agents for their treatment.

We identified nine SDH-deficient GISTs, as assessed by SDHB protein loss, all of which harboring mutations in one of the $S D H$ subunits (Table 1). Epigenetic inactivating mechanisms, such as allelic hemimethylation, can be hypothesized to explain the loss of SDHB protein in the three tumors of our series lacking a detectable second hit, bearing a heterozygous SHDx mutation; in fact, combined heterozygous mutation and hemimethylation have been reported in $S D H x$ (namely, $S D H C$ ) in some GISTs; moreover, SDHA-mutant GISTs which, despite the absence of a detectable second hit, nevertheless featured loss of SDHB expression have also been signaled [14]. Of note, our finding of five cases lacking germline $S D H x$ mutations out of $8 S D H x$-mutant SDHdeficient ones whose germline could be investigated could possibly support a relatively common occurrence of this GIST type in the absence of a syndromic predisposition.

In our series, all MGMT-methylated cases but one were detected in the pathogenetically heterogeneous group of GIST WT for KIT and PDGFRA, and SDH-deficient GISTs were significantly enriched in MGMT-methylated cases; noticeably, with the limitations due to the small number of cases considered, SDH-deficient GISTs featured the highest prevalence of MGMT methylation $(6 / 9,67 \%)$ when compared to the other pathogenetically characterized GIST subgroups of our series $(0 / 10,0 \%$, of PDGFRA-mutant GISTs; $1 / 15$, 7\%, of KIT-mutant GISTs; $1 / 5,20 \%$, of NF1-associated GISTs; Table 1). Additionally, we found an overall good correlation between MGMT methylation and MGMT protein expression, as assessed by IHC (the discrepancy found in a minority of cases could depend on DNA methylation levels not enough to inhibit protein expression on the one hand, or on mechanisms affecting protein expression alternative to DNA methylation, such as histone modifications or post-transcriptional regulation, on the other [40]). Thus, our results support the potential predisposition to respond to alkylating agents of a subset of the hitherto globally chemorefractory KIT/ PDGFRA WT GISTs, including their largest pathogenetically characterized subtype: SDH-deficient ones.

We do not know whether the MGMT methylation we observed in KIT/PDGFRA-WT GISTs, including those $\mathrm{SDH}$-deficient, is due to a mechanism specifically targeting MGMT or not (of note, this issue would not affect the potential therapeutic implications of the event). The DNA methylation described in SDH-deficient GISTs, caused by the impairment of the conversion of 5-methylcytosine to 5-hydroxymethylcytosine (required for DNA demethylation) due to the inhibition of TET DNA-hydroxylases secondary to succinate accumulation [3], could non-specifically contribute to the enrichment in MGMT-methylated cases we found among SDH-deficient GISTs. Nevertheless, since, as noted above, DNA methylation in these latter tumors is not randomly distributed [12-14], hitherto unknown gene-specific mechanisms regulating DNA methylation, possibly similar to that exerted by HOTAIR in some GISTs [41], are bound to flank the non-specific DNA methylation machinery described in SDH-deficient GISTs, and could affect MGMT also. Conversely, to the best of our knowledge, neither 
relatively high-DNA methylation levels, nor DNA methylation mechanisms, have been described in KIT/ PDGFRA-WT, SDH-competent GISTs, explaining the significant MGMT methylation we found in these tumors.

The cases of our series were arbitrarily assorted with the aim of investigating MGMT methylation in GIST subgroups differing in molecular trigger; consequently, rare GIST types such as SDH-deficient and NF1-related ones are overrepresented with respect to populationbased series. Nevertheless, considering the known epidemiology of GISTs [1], our finding of MGMT methylation substantially restricted to almost half of the investigated KIT/PDGFRA WT cases, even though these also were arbitrarily assorted in our series, supports a rare occurrence of this condition in GISTs, probably accounting for $\leq 5-10 \%$ of cases. To the best of our knowledge, only four papers have reported on MGMT methylation or expression in GISTs, employing heterogeneous methods. Two of them, both employing MS-specific PCR, report a relatively high fraction of $M G M T$-methylated GISTs, potentially challenging the concept that MGMT methylation is a relatively rare event in these tumors [15-17, 20]. However, one of these papers dealt exclusively with gastric cases [15], which are expectedly enriched in SDH-deficient GISTs, albeit presumably not at a level to justify per se the detected prevalence of MGMT-methylation (of note, SDH status was not investigated, coherently with the epoch of the study, which preceded the discovery of the role of SDH in GIST pathogenesis [42]). The paper by Saito and co-workers [16] overtly contrasts with our findings in terms of expected low prevalence of MGMT-methylated GISTs; nonetheless, all three of the three (100\%) reported cases WT for both KIT and PDGFRA featured a methylated MGMT. Noticeably, none of these works concerning MGMT methylation in GISTs was population-based.

Published data on the effectiveness of alkylating agents temozolomide and carmustine in GIST treatment, although apparently discouraging, do not stand definitively against a possible efficacy of alkylating agents restricted to a subset of the uncommon KIT/PDGFRA WT GISTs, including SDH-deficient ones. In fact, these results are based on a limited number of GIST patients recruited in past trials on sarcomas, without either selecting the genotype or investigating the SDH status of the tumors $[18-20]$. The latter event is again coherent with the epoch of the referred studies. Moreover, MGMT status was studied only in two of these tumors, significantly revealing spontaneous activity and, although $\mathrm{O}^{6}$-benzylguanine was administered with the aim of inhibiting this enzyme, the achievement of such an effect was not verified [20]. Finally, Choi response criteria, the most sensitive method for detecting GIST response to drugs [21], were not employed, once more coherently with the epoch of the referred papers. Thus, a possible effect of alkylating agents on a predisposed fraction of GISTs could have so far escaped detection since (1) we herein prove that MGMT methylation is substantially restricted to rare subsets of these tumors, implying the possibility that a few (or even none) of the limited number of GIST patients enrolled in the abovementioned trials could respond to the administered therapy, and (2) a possible response of some GISTs, bearing an inactivated MGMT, could have escaped detection, given the missed adoption of Choi criteria. This scenario could have led to erroneously abort further studies on alkylating agents in GISTs. As confirmation of our hypothesis, a phase 2 trial investigating a response to the drug temozolomide in metastatic SDH-deficient GIST to improve patient outcomes was recently launched and is ongoing (ClinicalTrials.gov/NCT03556384).

\section{Conclusions}

A subset of KIT/PDGFRA-WT GISTs, including their largest pathogenetically characterized subgroup (i.e., SDH-deficient GISTs), is preferentially MGMT-methylated. This finding fosters a reappraisal of alkylating agents for treating malignant cases occurring among these overall chemorefractory tumors.

\section{Additional files}

Additional file 1: Figure S1. Microphotograph showing a SDHdeficient GIST (case 43). (a) Tumor consisted of sheets of epithelioid cells with eosinophilic cytoplasm (scale bar: $15 \mu \mathrm{m}$ ). (b) Tumor cells lack cytoplasmic SDHB granular positivity, retained in non-neoplastic cells (notice the cytoplasmic labeling of smooth muscle cells, both in gastric muscularis propria - top left-and in a blood vessel wall-center-, or of scattered tumor infiltrating leukocytes and plasma cells) (scale bar: $15 \mu \mathrm{m}$ ). (TIF $16742 \mathrm{~kb}$ )

Additional file 2: Figure S2. Chromatogram showing the $\mathrm{SDHX}$ mutations found in GISTs (T) and in normal tissue $(N)$ in cases 42-48. Case 42: heterozygous SDHA exon 5 mutation in normal tissue and homozygous in GIST. Case 43: somatic heterozygous SDHA exons 1 and 13 mutations in GIST. Case 44: somatic heterozygous SDHA exon 9 mutation in GIST. Case 45: somatic homozygous SDHA exon 10 mutation in GIST. Case 46: somatic homozygous SDHB exon 6 mutation in GIST. Case 47: somatic heterozygous SDHC exon 4 mutation in GIST. Case 48: heterozygous SDHA exon 9 mutation in GIST (germline not tested). (SDHX mutations found in cases 40 and 41 have been previously reported-see ref. [26] of the main text). (PPTX $1802 \mathrm{~kb}$ )

\section{Abbreviations}

GIST: Gastrointestinal stromal tumor; MGMT: $0^{6}$-methylguanine DNA methyltransferase; PDGFRA: Platelet-derived growth factor receptor alpha; $\mathrm{SDH}$ : Succinate dehydrogenase

\section{Acknowledgements}

Not applicable.

\section{Funding}

This work was supported by grants from the Università Cattolica del Sacro Cuore (Linea D1 grants numbers R4124500212 and R4124500331 to RR, and 4124500389 to MM). 


\section{Availability of data and materials}

The datasets used and/or analyzed during the current study are available from the corresponding author on reasonable request.

\section{Authors' contributions}

RR conceived the study. RR, MMa, GR, MMi, SAn, AC, MP, and LML designed the experiments and analyzed the data. MMa, GR, TC, MMi, PL, FP, and DS performed and evaluated the experiments. AB, FR, GC, SAl, and RP performed surgery and provided essential materials. RR and GR wrote the manuscript. RR, MMa, and GR assembled the figures and tables. All authors critically revised the manuscript and approved its final version.

\section{Ethics approval and consent to participate}

The procedures followed were in accordance with the ethical standards of the local institutional committees on human experimentation and with the Helsinki declaration of 1975, as revised in 1983. The Institutional Review Boards of the participating institutions approved the study (Università Cattolica del Sacro Cuore, Fondazione Policlinico Universitario "A Gemelli" prot. n. 9421/18-15338/18-ID1969 and Azienda Ospedaliero-Universitaria di Bologna, Policlinico S.Orsola-Malpighi prot. n. 1258/2008). Informed consent to be included in the study was obtained from all patients.

\section{Consent for publication}

Not applicable

\section{Competing interests}

RR and AC have received speaker's honoraria from Novartis. For the remaining authors, none were declared.

\section{Publisher's Note}

Springer Nature remains neutral with regard to jurisdictional claims in published maps and institutional affiliations.

\section{Author details}

'Department of Pathology, Università Cattolica del Sacro Cuore, Largo F.Vito 1, 00168 Rome, Italy. ${ }^{2}$ UOC di Anatomia Patologica, Fondazione Policlinico Universitario "A. Gemelli" IRCCS, Largo A. Gemelli 8, 00168 Rome, Italy. ${ }^{3}$ Department of Pharmacy and Biotechnology, University of Bologna, via Massarenti 9, 40138 Bologna, Italy. ${ }^{4}$ Department of Pathology and Laboratory Medicine, Fondazione IRCCS Istituto Nazionale dei Tumori, Via Venezian, 20100 Milan, Italy. ${ }^{5}$ Pathology Unit, S.Orsola-Malpighi Hospital, University of Bologna, via Massarenti 9, 40138 Bologna, Italy. ${ }^{6}$ UOC di Chirurgia Generale, Fondazione Policlinico Universitario "A. Gemelli" IRCCS, Largo A. Gemelli 8, 00168 Rome, Italy. ${ }^{7}$ UOC di Chirurgia Digestiva, Fondazione Policlinico Universitario "A. Gemelli" IRCCS, Largo A. Gemelli 8, 00168 Rome, Italy. ${ }^{8}$ Department of Surgery, Università Cattolica del Sacro Cuore, Largo F. Vito 1, 00168 Rome, Italy. ${ }^{9}$ UOC di Chirurgia Generale ed Epato-Biliare, Fondazione Policlinico Universitario "A. Gemelli" IRCCS, Largo A. Gemelli 8, 00168 Rome, Italy. ${ }^{10}$ Department of Internal Medicine, Università Cattolica del Sacro Cuore, Largo F. Vito 1, 00168 Rome, Italy. ${ }^{11}$ UOC di Oncologia Medica, Fondazione Policlinico Universitario "A. Gemelli" IRCCS, Largo A. Gemelli 8, 00168 Rome, Italy. ${ }^{12}$ Department of Experimental, Diagnostic and Specialty Medicine, University of Bologna, via Massarenti 9, 40138 Bologna, Italy.

\section{Received: 7 August 2018 Accepted: 3 December 2018}

Published online: 07 January 2019

\section{References}

1. Ricci R, Dei Tos AP, Rindi G. GISTogram: a graphic presentation of the growing GIST complexity. Virchows Arch. 2013;463:481-7.

2. Biasco G, Velo D, Angriman I, Astorino M, Baldan A, Baseggio M, et al. Gastrointestinal stromal tumors: report of an audit and review of the literature. Eur J Cancer Prev. 2009;18:106-16.

3. Ricci R. Syndromic gastrointestinal stromal tumors. Hered Cancer Clin Pract. 2016;14:15.

4. Weldon CB, Madenci AL, Boikos SA, Janeway KA, George S, von Mehren M, et al. Surgical management of wild-type gastrointestinal stromal tumors: a report from the National Institutes of Health Pediatric and Wildtype GIST Clinic. J Clin Oncol. 2017;10(35):523-8.

5. Boikos SA, Pappo AS, Killian JK, LaQuaglia MP, Weldon CB, George S, et al. Molecular subtypes of KIT/PDGFRA wild-type gastrointestinal stromal tumors: a report from the National Institutes of Health Gastrointestinal Stromal Tumor Clinic. JAMA Oncol. 2016;2:922-8.

6. Ben-Ami E, Barysauskas CM, von Mehren M, Heinrich MC, Corless CL, Butrynski JE, et al. Long-term follow-up results of the multicenter phase II trial of regorafenib in patients with metastatic and/or unresectable Gl stromal tumor after failure of standard tyrosine kinase inhibitor therapy. Ann Oncol. 2016;27:1794-9.

7. Pantaleo MA. Good survival outcome of metastatic SDH-deficient gastrointestinal stromal tumours harbouring SDHA mutations. Genet Med. 2015:17:391-5.

8. Esteller M, Garcia-Foncillas J, Andion E, Goodman SN, Hidalgo OF, Vanaclocha $V$, et al. Inactivation of the DNA-repair gene MGMT and the clinical response of gliomas to alkylating agents. N Engl J Med. 2000; 343:1350-4.

9. Inno A, Fanetti G, Di Bartolomeo M, Gori S, Maggi C, Cirillo M, et al. Role of MGMT as biomarker in colorectal cancer. World J Clin Cases. 2014;2:835-9.

10. Esteller M, Gaidano G, Goodman SN, Zagonel V, Capello D, Botto B, et al. Hypermethylation of the DNA repair gene O(6)-methylguanine DNA methyltransferase and survival of patients with diffuse large B-cell lymphoma. J Natl Cancer Inst. 2002;94:26-32.

11. Hegi ME, Diserens AC, Gorlia T, Hamou MF, de Tribolet N, Weller M, et al. MGMT gene silencing and benefit from temozolomide in glioblastoma. N Engl J Med. 2005;352:997-1003.

12. Haller F, Moskalev EA, Faucz FR, Barthelmess S, Wiemann S, Bieg M, et al. Aberrant DNA hypermethylation of SDHC: a novel mechanism of tumor development in Carney triad. Endocr Relat Cancer. 2014;21:567-77.

13. Killian JK, Kim SY, Miettinen M, Smith C, Merino M, Tsokos M, et al. Succinate dehydrogenase mutation underlies global epigenomic divergence in gastrointestinal stromal tumor. Cancer Discov. 2013;3:648-57.

14. Killian JK, Miettinen M, Walker RL, Wang Y, Zhu YJ, Waterfall JJ, et al. Recurrent epimutation of SDHC in gastrointestinal stromal tumors. Sci Transl Med. 2014;6:268ra177.

15. House MG, Guo M, Efron DT, Lillemoe KD, Cameron JL, Syphard JE, et al. Tumor suppressor gene hypermethylation as a predictor of gastric stromal tumor behavior. J Gastrointest Surg. 2003;7:1004-14.

16. Saito K, Sakurai S, Sano T, Sakamoto K, Asao T, Hosoya Y, et al. Aberrant methylation status of known methylation-sensitive $\mathrm{CpG}$ islands in gastrointestinal stromal tumors without any correlation to the state of c-kit and PDGFRA gene mutations and their malignancy. Cancer Sci. 2008;99:253-9.

17. Okamoto Y, Sawaki A, Ito S, Nishida T, Takahashi T, Toyota M, et al. Aberrant DNA methylation associated with aggressiveness of gastrointestinal stromal tumour. Gut. 2012;61:392-401.

18. Trent JC, Beach J, Burgess MA, Papadopolous N, Chen LL, Benjamin RS, et al. A two-arm phase II study of temozolomide in patients with advanced gastrointestinal stromal tumors and other soft tissue sarcomas. Cancer. 2003:98:2693-9.

19. Garcia del Muro X, Lopez-Pousa A, Martin J, Buesa JM, Martinez-Trufero J, Casado A, et al. A phase II trial of temozolomide as a 6-week, continuous, oral schedule in patients with advanced soft tissue sarcoma: a study by the Spanish Group for Research on Sarcomas. Cancer. 2005;104:1706-12.

20. Ryan CW, Dolan ME, Brockstein BB, McLendon R, Delaney SM, Samuels BL, et al. A phase II trial of O6-benzylguanine and carmustine in patients with advanced soft tissue sarcoma. Cancer Chemother Pharmacol. 2006;58:634-9.

21. Benjamin RS, Choi H, Macapinlac HA, Burgess MA, Patel SR, Chen LL, et al. We should desist using RECIST, at least in GIST. J Clin Oncol. 2007;25:1760-4.

22. Ricci R, Martini M, Cenci T, Carbone A, Lanza P, Biondi A, et al. PDGFRAmutant syndrome. Mod Pathol. 2015;28:954-64.

23. Basso M, Strippoli A, Orlandi A, Martini M, Calegari MA, Schinzari G, et al. KRAS mutational status affects oxaliplatin-based chemotherapy independently from basal mRNA ERCC-1 expression in metastatic colorectal cancer patients. Br J Cancer. 2013;108:115-20.

24. Rossi ED, Martini M, Capodimonti S, Straccia P, Cenci T, Lombardi CP, et al. Diagnostic and prognostic value of immunocytochemistry and BRAF mutation analysis on liquid-based biopsies of thyroid neoplasms suspicious for carcinoma. Eur J Endocrinol. 2013;168:853-9.

25. Miranda C, Nucifora M, Molinari F, Conca E, Anania MC, Bordoni A, et al. KRAS and BRAF mutations predict primary resistance to imatinib in gastrointestinal stromal tumors. Clin Cancer Res. 2012;18:1769-76.

26. Pantaleo MA, Astolfi A, Indio V, Moore R, Thiessen N, Heinrich MC, et al. SDHA loss-of-function mutations in KIT-PDGFRA wild-type gastrointestinal 
stromal tumors identified by massively parallel sequencing. J Natl Cancer Inst. 2011;103:983-7.

27. Ricci R, Martini M, Cenci T, Riccioni ME, Maria G, Cassano A, et al. Divergent gastrointestinal stromal tumors in syndromic settings. Cancer Genet. 2016;209:354-8.

28. Miettinen M, Lasota J. Succinate dehydrogenase deficient gastrointestinal stromal tumors (GISTs) - a review. Int J Biochem Cell Biol. 2014;53:514-9.

29. Pantaleo MA, Astolfi A, Urbini M, Nannini M, Paterini P, Indio V, et al. Analysis of all subunits, SDHA, SDHB, SDHC, SDHD, of the succinate dehydrogenase complex in KIT/PDGFRA wild-type GIST. Eur J Hum Genet. 2014;22:32-9.

30. Ricci R, Arena V, Castri F, Martini M, Maggiano N, Murazio M, et al. Role of p16/INK4a in gastrointestinal stromal tumor progression. Am J Clin Pathol. 2004;122:35-43.

31. Martini M, Pallini R, Luongo G, Cenci T, Lucantoni C, Larocca LM. Prognostic relevance of SOCS3 hypermethylation in patients with glioblastoma multiforme. Int J Cancer. 2008;123:2955-60.

32. Rodriguez FJ, Thibodeau SN, Jenkins RB, Schowalter KV, Caron BL, O'Neill BP, et al. MGMT immunohistochemical expression and promoter methylation in human glioblastoma. Appl Immunohistochem Mol Morphol. 2008;16(1):59-65.

33. Miettinen M, Wang ZF, Sarlomo-Rikala M, Osuch C, Rutkowski P, Lasota J. Succinate dehydrogenase-deficient GISTs: a clinicopathologic, immunohistochemical, and molecular genetic study of 66 gastric GISTs with predilection to young age. Am J Surg Pathol. 2011;35(11):1712-21.

34. Boikos SA, Xekouki P, Fumagalli E, Faucz FR, Raygada M, Szarek E, et al. Carney triad can be (rarely) associated with germline succinate dehydrogenase defects. Eur J Hum Genet. 2016;24:569-73.

35. Huss S, Elges S, Trautmann M, Sperveslage J, Hartmann W, Wardelmann E. Classification of KIT/PDGFRA wild-type gastrointestinal stromal tumors: implications for therapy. Expert Rev Anticancer Ther. 2015;15(6):623-8.

36. Sapi Z, Fule T, Hajdu M, Matolcsy A, Moskovszky L, Mark A, et al. The activated targets of mTOR signaling pathway are characteristic for PDGFRA mutant and wild-type rather than KIT mutant GISTs. Diagn Mol Pathol. 2011;20:22-33.

37. Mason EF, Hornick JL. Succinate dehydrogenase deficiency is associated with decreased 5-hydroxymethylcytosine production in gastrointestinal stromal tumors: implications for mechanisms of tumorigenesis. Mod Pathol. 2013;26(11):1492-7.

38. Kim SY, Janeway K, Pappo A. Pediatric and wild-type gastrointestinal stromal tumor: new therapeutic approaches. Curr Opin Oncol. 2010;22:347-50.

39. Vadakara J, von Mehren M. Gastrointestinal stromal tumors: management of metastatic disease and emerging therapies. Hematol Oncol Clin North Am. 2013:27:905-20

40. Wang L, Li Z, Liu C, Chen L, Liu L, Hu Z, et al. Comparative assessment of three methods to analyze MGMT methylation status in a series of 350 gliomas and gangliogliomas. Pathol Res Pract. 2017;213(12):1489-93.

41. Bure I, Geer S, Knopf J, Roas M, Henze S, Strobel P, et al. Long noncoding RNA HOTAIR is upregulated in an aggressive subgroup of gastrointestinal stromal tumors (GIST) and mediates the establishment of gene-specific DNA methylation patterns. Genes Chromosomes Cancer. 2018;57(11):584-97.

42. McWhinney SR, Pasini B, Stratakis CA, International Carney T, Carney-Stratakis Syndrome C. Familial gastrointestinal stromal tumors and germ-line mutations. N Engl J Med. 2007;357:1054-6.

Ready to submit your research? Choose BMC and benefit from:

- fast, convenient online submission

- thorough peer review by experienced researchers in your field

- rapid publication on acceptance

- support for research data, including large and complex data types

- gold Open Access which fosters wider collaboration and increased citations

- maximum visibility for your research: over $100 \mathrm{M}$ website views per year

At BMC, research is always in progress.

Learn more biomedcentral.com/submissions 